\title{
Robust Stability for Stochastic Hopfield Neural Networks with Time Delays
}

\author{
Zidong Wang, Huisheng Shu, Jian'an Fang, and Xiaohui Liu
}

\begin{abstract}
In this paper, the asymptotic stability analysis problem is considered for a class of uncertain stochastic neural networks with time delays and parameter uncertainties. The delays are time-invariant, and the uncertainties are normbounded that enter into all the network parameters. The aim of this paper is to establish easily verifiable conditions under which the delayed neural network is robustly asymptotically stable in the mean square for all admissible parameter uncertainties. By employing a Lyapunov-Krasovskii functional and conducting the stochastic analysis, a linear matrix inequality (LMI) approach is developed to derive the stability criteria. The proposed criteria can be checked readily by using some standard numerical packages, and no tuning of parameters is required. Examples are provided to demonstrate the effectiveness and applicability of the proposed criteria.
\end{abstract}

\section{Keywords}

Hopfield neural networks; Uncertain systems; Stochastic systems; Time delays; Lyapunov-Krasovskii functional; Global asymptotic stability; Linear matrix inequality.

\section{INTRODUCTION}

Since the seminal work for Hopfield neural networks in [16], [17], the past two decades have witnessed the successful applications of Hopfield neural networks in many areas such as combinatorial optimization, signal processing and pattern recognition, see e.g. [21], [22], [30]. Recently, it has been realized that the axonal signal transmission delays often occur in various neural networks, and may cause undesirable dynamic network behaviors such as oscillation and instability. Consequently, the stability analysis problems for delayed neural networks have gained considerable research attention. Up to now, a great deal of results have been reported in the literature, see e.g. [1], [6], [7], [9], [10], [20], [27] and references therein, where the delay type can be constant, time-varying, or distributed, and the stability criteria can be delay-dependent or delay-independent.

In real nervous systems, the synaptic transmission is a noisy process brought on by random fluctuations from the release of neurotransmitters and other probabilistic causes. It has also been known that a neural network could be stabilized or destabilized by certain stochastic inputs [3]. Hence, the stability analysis problem for stochastic neural networks becomes increasingly significant, and some results related to this problem have recently been published, see e.g. [3], [18], [19], [24]. On the other hand, the connection weights of the neurons depend on certain resistance and capacitance values that include uncertainties (modeling errors). When

This work was supported in part by the Engineering and Physical Sciences Research Council (EPSRC) of the U.K. under Grant GR/S27658/01, the Nuffield Foundation of the U.K. under Grant NAL/00630/G, and the Alexander von Humboldt Foundation of Germany.

Z. Wang is with the Department of Information Systems and Computing, Brunel University, Uxbridge, Middlesex, UB8 3PH, U.K., and is also with the School of Information Sciences and Technology, Donghua University, Shanghai 200051, China. Email: Zidong.Wang@brunel.ac.uk, Fax: ++44/1895 251686.

H. Shu is with the School of Sciences, Donghua University, Shanghai 200051, China.

J. Fang is with the School of Information Sciences and Technology, Donghua University, Shanghai 200051, China.

$\mathrm{X}$. Liu is with the Department of Information Systems and Computing, Brunel University, Uxbridge, Middlesex, UB8 3PH, U.K. 
modeling neural networks, the parameter uncertainties (also called variations or fluctuations) should be taken into account, and therefore the problem of robust stability analysis for neural networks emerges as a research topic of primary importance, see e.g. [2], [8], [11], [29]. It should be pointed out that, in most existing literature, the stochastic analysis issue and stability robustness issue have been treated separately. To the best of the authors' knowledge, the robust stability analysis problem for stochastic Hopfield neural networks with time delays has not been fully investigated, and remains important and challenging.

In this paper, we deal with the global robust stability analysis problem for a class of stochastic Hopfield neural networks with time-delays. By utilizing a Lyapunov-Krasovskii functional and using the well-known $S$ procedure, we convert the addressed stability analysis problem into a convex optimization problem. Different from the commonly used matrix norm theories (such as the $M$-matrix method), a unified linear matrix inequality (LMI) approach is developed to establish sufficient conditions for the neural networks to be robustly, globally, asymptotically stable. Note that LMIs can be easily solved by using the Matlab LMI toolbox, and no tuning of parameters is required [4]. Two numerical examples are provided to show the usefulness of the proposed global stability condition.

Notations: The notations are quite standard. Throughout this paper, $\mathbb{R}^{n}$ and $\mathbb{R}^{n \times m}$ denote, respectively, the $n$-dimensional Euclidean space and the set of all $n \times m$ real matrices. The superscript " $T$ " denotes matrix transposition and the notation $X \geq Y$ (respectively, $X>Y$ ) where $X$ and $Y$ are symmetric matrices, means that $X-Y$ is positive semidefinite (respectively, positive definite). $I_{n}$ is the $n \times n$ identity matrix. $|\cdot|$ is the Euclidean norm in $\mathbb{R}^{n}$. If $A$ is a matrix, denote by $\|A\|$ its operator norm, i.e., $\|A\|=\sup \{|A x|:|x|=1\}=$ $\sqrt{\lambda_{\max }\left(A^{T} A\right)}$ where $\lambda_{\max }(\cdot)$ (respectively, $\lambda_{\min }(\cdot)$ ) means the largest (respectively, smallest) eigenvalue of $A$. $l_{2}[0, \infty]$ is the space of square integrable vector. Moreover, let $\left(\Omega, \mathcal{F},\left\{\mathcal{F}_{t}\right\}_{t \geq 0}, P\right)$ be a complete probability space with a filtration $\left\{\mathcal{F}_{t}\right\}_{t \geq 0}$ satisfying the usual conditions (i.e., the filtration contains all $P$-null sets and is right continuous $)$. Denote by $L_{\mathcal{F}_{0}}^{p}\left([-h, 0] ; \mathbb{R}^{n}\right)$ the family of all $\mathcal{F}_{0}$-measurable $C\left([-h, 0] ; \mathbb{R}^{n}\right)$-valued random variables $\xi=\{\xi(\theta):-h \leq \theta \leq 0\}$ such that $\sup _{-h \leq \theta \leq 0} \mathbb{E}|\xi(\theta)|^{p}<\infty$ where $\mathbb{E}\{\cdot\}$ stands for the mathematical expectation operator with respect to the given probability measure $P$. The shorthand $\operatorname{diag}\left\{M_{1}, M_{2}, \cdots, M_{N}\right\}$ denotes a block diagonal matrix with diagonal blocks being the matrices $M_{1}, M_{2}, \cdots, M_{N}$. Sometimes, the arguments of a function or a matrix will be omitted in the analysis when no confusion can arise.

\section{Problem Formulation}

In the past few years, the dynamical behavior (especially the stability) of the Hopfield neural networks with time delays have been intensively studied, and many stability criteria have been proposed, see e.g. [2], [7], [8], [9], [10], [27], [31]. The Hopfield neural network with time delays can be described by the following model:

$$
\dot{u}(t)=-A u(t)+W g(u(t-h))+V
$$

where $u(t)=\left[u_{1}(t), u_{2}(t), \cdots, u_{n}(t)\right]^{T} \in \mathbb{R}^{n}$ is the state vector associated with the $n$ neurons, the diagonal matrix $A=\operatorname{diag}\left(a_{1}, a_{2}, \cdots, a_{n}\right)$ has positive entries $a_{i}>0$. The matrices $W=\left(w_{i j}\right)_{n \times n}$ is the connection weight matrix. $g(u(t))=\left[g_{1}\left(u_{1}(t)\right), g_{2}\left(u_{2}(t)\right), \cdots, g_{n}\left(u_{n}(t)\right)\right]^{T}$ denotes the neuron activation function with $g(0)=0$, and $V=\left[V_{1}, V_{2}, \cdots, V_{n}\right]^{T}$ is a constant external input vector. The scalar $h>0$ denotes the discrete time delay.

The following assumption is made on the neuron activation function.

Assumption 1: The neuron activation function $g(\cdot)$ in (1) satisfies the following Lipschitz condition

$$
|g(x)-g(y)| \leq|G(x-y)|, \quad \forall x, y \in \mathbb{R}
$$


where $G \in \mathbb{R}^{n \times n}$ is a known constant matrix.

Remark 1: The activation functions are typically assumed to be continuous, differentiable, monotonically increasing and bounded, such as the functions of sigmoid type. These conditions are no longer needed in this paper. Instead, only the Lipschitz condition is imposed in Assumption 1. Note that the type of activation functions in (2) have already been used in numerous papers, see [9] and references therein.

Let $u^{*}$ be the equilibrium point of (1). For the purpose of simplicity, we can shift the intended equilibrium $u^{*}$ to the origin by letting $x=u-u^{*}$, and then the system (1) can be transformed into:

$$
\dot{x}(t)=-A x(t)+W l(x(t-h)),
$$

where $x(t)=\left[x_{1}(t), x_{2}(t), \cdots, x_{n}(t)\right]^{T} \in \mathbb{R}^{n}$ is the state vector of the transformed system. It follows from (2) that the transformed neuron activation function $l(x)=g\left(x+u^{*}\right)-g\left(u^{*}\right)$ satisfies

$$
|l(x)| \leq|G x|,
$$

where $G \in \mathbb{R}^{n \times n}$ is specified in (2).

As mentioned previously, it is often the case in practice that the network parameters may contain uncertainties due to modeling errors, and the neural network is disturbed by environmental noises that affect the stability of the equilibrium. In this paper, the Hopfield neural network with parameter uncertainties and stochastic perturbations is described as follows:

$$
d x(t)=[-(A+\Delta A) x(t)+(W+\Delta W) l(x(t-h))] d t+[(\Delta C) x(t)+(\Delta D) x(t-h)] d w(t),
$$

where $w(t)=\left[w_{1}(t), w_{2}(t), \cdots, x_{m}(t)\right]^{T} \in \mathbb{R}^{m}$ is a Brownian motion defined on $\left(\Omega, \mathcal{F},\left\{\mathcal{F}_{t}\right\}_{t \geq 0}, P\right)$, and the matrices $\Delta A, \Delta W, \Delta C$ and $\Delta D$ are of the following structure

$$
\left[\begin{array}{llll}
\Delta A & \Delta W & \Delta C & \Delta D
\end{array}\right]=M F\left[\begin{array}{llll}
N_{1} & N_{2} & N_{3} & N_{4}
\end{array}\right]
$$

where $A, W, M, N_{i}(i=1,2,3,4)$ are known real constant matrices with appropriate dimensions, and the uncertain matrix $F$, which may be time-varying, is unknown and satisfies

$$
F^{T} F \leq I .
$$

Remark 2: The parameter uncertainty structure as in (6)-(7) has been widely exploited in the problems of robust control and robust filtering of uncertain systems (see e.g., [25], [26] and the references therein). Many practical systems possess parameter uncertainties which can be either exactly modeled or overbounded by $(7)$. Observe that the unknown matrix $F$ in (6) can even be allowed to be state-dependent, i.e., $F(t)=F(t, x(t))$, as long as (7) is satisfied. On the other hand, the stochastic disturbance term, $[(\Delta C) x(t)+(\Delta D) x(t-h)] d w(t)$, can be viewed as stochastic perturbations on the neuron states and delayed neuron states.

Let $x(t ; \xi)$ denote the state trajectory of the neural network (5) from the initial data $x(\theta)=\xi(\theta)$ on $-h \leq \theta \leq 0$ in $L_{\mathcal{F}_{0}}^{2}\left([-h, 0] ; \mathbb{R}^{n}\right)$. It can be easily seen that the system $(5)$ admits a trivial solution $x(t ; 0) \equiv 0$ corresponding to the initial data $\xi=0$, see [5], [15].

We are now ready to introduce the notion of robust global asymptotic stability for the stochastic neural network (5) with parameter uncertainties and time-delays.

Definition 1: For the neural network (5) and every $\xi \in L_{\mathcal{F}_{0}}^{2}\left([-h, 0] ; \mathbb{R}^{n}\right)$, the trivial solution (equilibrium point) is robustly, globally, asymptotically stable in the mean square if, for all admissible uncertainties satisfying (7), the following holds:

$$
\lim _{t \rightarrow \infty} \mathbb{E}|x(t ; \xi)|^{2}=0
$$


The main purpose of this paper is to establish LMI-based stability criteria under which the global robust asymptotic stability of the uncertain stochastic delayed neural network (5) can be tested conveniently by using the Matlab LMI toolbox.

\section{MAIN RESUltS AND PROOFS}

The following lemmas will be repeatedly used in deriving the desired LMI-based stability criteria.

Lemma 1: Let $x \in \mathbb{R}^{n}, y \in \mathbb{R}^{n}$ and $\varepsilon>0$. Then we have $x^{T} y+y^{T} x \leq \varepsilon x^{T} x+\varepsilon^{-1} y^{T} y$.

Proof: The proof follows from the inequality $\left(\varepsilon^{1 / 2} x-\varepsilon^{-1 / 2} y\right)^{T}\left(\varepsilon^{1 / 2} x-\varepsilon^{-1 / 2} y\right) \geq 0$ immediately.

Lemma 2: [25] Let $A, D, E, F$ and $P$ be real matrices of appropriate dimensions with $P>0$ and $F$ satisfying $F^{T} F \leq I$. Then for any scalar $\varepsilon>0$ satisfying $P^{-1}-\varepsilon^{-1} D D^{T}>0$, we have

$$
(A+D F E)^{T} P(A+D F E) \leq A^{T}\left(P^{-1}-\varepsilon^{-1} D D^{T}\right)^{-1} A+\varepsilon E^{T} E .
$$

Lemma 3: [4] Given constant matrices $\Sigma_{1}, \Sigma_{2}, \Sigma_{3}$ where $\Sigma_{1}=\Sigma_{1}^{T}$ and $0<\Sigma_{2}=\Sigma_{2}^{T}$, then

$$
\Sigma_{1}+\Sigma_{3}^{T} \Sigma_{2}^{-1} \Sigma_{3}<0
$$

if and only if

$$
\left[\begin{array}{cc}
\Sigma_{1} & \Sigma_{3}^{T} \\
\Sigma_{3} & -\Sigma_{2}
\end{array}\right]<0, \quad \text { or } \quad\left[\begin{array}{cc}
-\Sigma_{2} & \Sigma_{3} \\
\Sigma_{3}^{T} & \Sigma_{1}
\end{array}\right]<0 .
$$

Lemma 4: [14] For any positive definite matrix $M>0$, scalar $\gamma>0$, vector function $\omega:[0, \gamma] \rightarrow \mathbb{R}^{n}$ such that the integrations concerned are well defined, the following inequality holds:

$$
\left(\int_{0}^{\gamma} \omega(s) d s\right)^{T} M\left(\int_{0}^{\gamma} \omega(s) d s\right) \leq \gamma\left(\int_{0}^{\gamma} \omega^{T}(s) M \omega(s) d s\right)
$$

For the sake of presentation simplicity, we denote:

$$
\begin{aligned}
& \Omega_{1}:=-A P-P A+Q_{1}+h Q_{2}+\mu_{4} N_{3}^{T} N_{3}, \\
& \Omega_{2}:=\left(\varepsilon_{1}+\varepsilon_{3}\right) P M M^{T} P+\varepsilon_{1}^{-1} N_{1}^{T} N_{1}+\varepsilon_{2} P W W^{T} P, \\
& R_{1}:=\left[\begin{array}{llll}
\varepsilon_{1}^{1 / 2} P M & \varepsilon_{1}^{-1 / 2} N_{1}^{T} & \varepsilon_{2}^{1 / 2} P W & \varepsilon_{3}^{1 / 2} P M
\end{array}\right], \\
& R_{2}:=\left[\begin{array}{ll}
\varepsilon_{2}^{-1 / 2} G^{T} & \varepsilon_{3}^{-1 / 2} \lambda_{\max }^{1 / 2}\left(N_{2}^{T} N_{2}\right) G^{T}
\end{array}\right], \\
& \Xi=-Q_{1}+\varepsilon_{2}^{-1} G^{T} G+\varepsilon_{3}^{-1} \lambda_{\max }\left(N_{2}^{T} N_{2}\right) G^{T} G+\varepsilon_{4} N_{4}^{T} N_{4} .
\end{aligned}
$$

The main theorem given below shows that the stability criteria can be expressed in terms of the feasibility of two linear matrix inequalities.

Theorem 1: If there exist positive scalars $\mu_{i}>0(i=1,2,3,4)$ and positive definite matrices $P=P^{T}>0$, 
$Q_{1}=Q_{1}^{T}>0$ and $Q_{2}=Q_{2}^{T}>0$ such that the following two linear matrix inequalities

$$
\begin{aligned}
& {\left[\begin{array}{cc}
-P & P M \\
M^{T} P & -\mu_{4} I
\end{array}\right]<0,} \\
& {\left[\begin{array}{cccccccc}
\Omega_{1} & \mu_{4} N_{4}^{T} N_{3} & P M & \mu_{1} N_{1}^{T} & P W & P M & 0 & 0 \\
\mu_{4} N_{3}^{T} N_{4} & -Q_{1}+\mu_{4} N_{4}^{T} N_{4} & 0 & 0 & 0 & 0 & \mu_{2} G^{T} & \mu_{3} \lambda_{\max }^{1 / 2}\left(N_{2}^{T} N_{2}\right) G^{T} \\
M^{T} P & 0 & -\mu_{1} I & 0 & 0 & 0 & 0 & 0 \\
\mu_{1} N_{1} & 0 & 0 & -\mu_{1} I & 0 & 0 & 0 & 0 \\
W^{T} P & 0 & 0 & 0 & -\mu_{2} I & 0 & 0 & 0 \\
M^{T} P & 0 & 0 & 0 & 0 & -\mu_{3} I & 0 & 0 \\
0 & \mu_{2} G & 0 & 0 & 0 & 0 & -\mu_{2} I & 0 \\
0 & \mu_{3} \lambda_{\max }^{1 / 2}\left(N_{2}^{T} N_{2}\right) G & 0 & 0 & 0 & 0 & 0 & -\mu_{3} I
\end{array}\right]<0}
\end{aligned}
$$

hold where $\Omega_{1}$ is defined in (9), then the dynamics of the neural network (5) is robustly, globally, asymptotically stable in the mean square.

Proof: First of all, denote

$$
\mu_{1}=\varepsilon_{1}^{-1}, \quad \mu_{2}=\varepsilon_{2}^{-1}, \quad \mu_{3}=\varepsilon_{3}^{-1}, \quad \mu_{4}=\varepsilon_{4} .
$$

Pre- and post-multiplying the inequality (15) by the block-diagonal matrix

$$
\operatorname{diag}\left\{I, I, \varepsilon_{1}^{1 / 2} I, \varepsilon_{1}^{1 / 2} I, \varepsilon_{2}^{1 / 2} I, \varepsilon_{3}^{1 / 2} I, \varepsilon_{2}^{1 / 2} I, \varepsilon_{3}^{1 / 2} I\right\}
$$

yield

$\left[\begin{array}{cccccccc}\Omega_{1} & \varepsilon_{4} N_{4}^{T} N_{3} & \varepsilon_{1}^{1 / 2} P M & \varepsilon_{1}^{-1 / 2} N_{1}^{T} & \varepsilon_{2}^{1 / 2} P W & \varepsilon_{3}^{1 / 2} P M & 0 & 0 \\ \varepsilon_{4} N_{3}^{T} N_{4} & -Q_{1}+\varepsilon_{4} N_{4}^{T} N_{4} & 0 & 0 & 0 & 0 & \varepsilon_{2}^{-1 / 2} G^{T} & \varepsilon_{3}^{-1 / 2} \lambda_{\max }^{1 / 2}\left(N_{2}^{T} N_{2}\right) G^{T} \\ \varepsilon_{1}^{1 / 2} M^{T} P & 0 & -I & 0 & 0 & 0 & 0 & 0 \\ \varepsilon_{1}^{-1 / 2} N_{1} & 0 & 0 & -I & 0 & 0 & 0 & 0 \\ \varepsilon_{2}^{1 / 2} W^{T} P & 0 & 0 & 0 & -I & 0 & 0 & 0 \\ \varepsilon_{3}^{1 / 2} M^{T} P & 0 & 0 & 0 & 0 & -I & 0 & 0 \\ 0 & \varepsilon_{2}^{-1 / 2} G & 0 & 0 & 0 & 0 & -I & 0 \\ 0 & \varepsilon_{3}^{-1 / 2} \lambda_{\max }^{1 / 2}\left(N_{2}^{T} N_{2}\right) G & 0 & 0 & 0 & 0 & 0 & -I\end{array}\right]<0$

or

$$
\left[\begin{array}{cc}
\Sigma_{1} & \Sigma_{3}^{T} \\
\Sigma_{3} & -\Sigma_{2}
\end{array}\right]<0
$$

where

$$
\Sigma_{1}:=\left[\begin{array}{cc}
\Omega_{1} & \varepsilon_{4} N_{4}^{T} N_{3} \\
\varepsilon_{4} N_{3}^{T} N_{4} & -Q_{1}+\varepsilon_{4} N_{4}^{T} N_{4}
\end{array}\right], \quad \Sigma_{3}:=\left[\begin{array}{cc}
R_{1}^{T} & 0 \\
0 & R_{2}^{T}
\end{array}\right], \quad \Sigma_{2}:=I,
$$

and $R_{1}$ and $R_{2}$ are defined in (11) and (12), respectively.

It follows from the Schur Complement Lemma (Lemma 3) that (17) holds if and only if

$$
\Sigma_{1}+\Sigma_{3}^{T} \Sigma_{2}^{-1} \Sigma_{3}<0
$$


or

$$
\left[\begin{array}{cc}
\Omega_{1}+\Omega_{2} & \varepsilon_{4} N_{4}^{T} N_{3} \\
\varepsilon_{4} N_{3}^{T} N_{4} & \Xi
\end{array}\right]<0,
$$

where $\Omega_{1}, \Omega_{2}$ and $\Xi$ are defined in (9), (10) and (13), respectively.

Summing up the results obtained so far, we conclude that, from the condition (15) of Theorem 1, there exist positive scalars $\varepsilon_{i}>0(i=1,2,3,4)$ and positive definite matrices $P=P^{T}>0, Q_{1}=Q_{1}^{T}>0$ and $Q_{2}=Q_{2}^{T}>0$ such that (18) is true. Also, it follows immediately from (4) that

$$
l^{T}(x) l(x) \leq|G x|^{2}=x^{T} G^{T} G x .
$$

Define a Lyapunov-Krasovskii functional candidate $V(t, x(t)) \in \mathcal{C}^{2,1}\left(\mathbb{R}^{+} \times \mathbb{R}^{n} ; \mathbb{R}^{+}\right)$by

$$
V(t, x(t))=x^{T}(t) P x(t)+\int_{t-h}^{t} x^{T}(s) Q_{1} x(s) d s+\int_{-h}^{0} \int_{t+s}^{t} x^{T}(\eta) Q_{2} x^{T}(\eta) d \eta d s .
$$

By Itô's differential formula (see, e.g., [12]), the stochastic derivative of $V(t, x(t))$ along (5) can be obtained as follows:

$$
\begin{aligned}
d V(t, x(t))= & \left\{x^{T}(t)\left(-A P-P A+Q_{1}+h Q_{2}\right) x(t)-2 x^{T}(t) P(\Delta A) x(t)\right. \\
& +2 x^{T}(t) P W l(x(t-h))+2 x^{T}(t) P(\Delta W) l(x(t-h)) \\
& +[(\Delta C) x(t)+(\Delta D) x(t-h)]^{T} P[(\Delta C) x(t)+(\Delta D) x(t-h)] \\
& \left.-x^{T}(t-h) Q_{1} x(t-h)-\int_{t-h}^{t} x^{T}(s) Q_{2} x(s) d s\right\} d t \\
& +\left\{2 x^{T}(t) P[(\Delta C) x(t)+(\Delta D) x(t-h)]\right\} d w(t) .
\end{aligned}
$$

For the positive scalars $\varepsilon_{1}>0, \varepsilon_{2}>0, \varepsilon_{3}>0$, it follows from Lemma 1 , the fact $F^{T} F \leq I$ and the relation (19) that

$$
\begin{aligned}
-2 x^{T}(t) P(\Delta A) x(t) & =-2 x^{T}(t) P M F N_{1} x(t) \\
& \leq \varepsilon_{1} x^{T}(t) P M M^{T} P x(t)+\varepsilon_{1}^{-1} x^{T}(t) N_{1}^{T} F^{T} F N_{1} x(t) \\
& \leq x^{T}(t)\left(\varepsilon_{1} P M M^{T} P+\varepsilon_{1}^{-1} N_{1}^{T} N_{1}\right) x(t), \\
2 x^{T}(t) P W l(x(t-h)) & \leq \varepsilon_{2} x^{T}(t) P W W^{T} P x(t)+\varepsilon_{2}^{-1} l^{T}(x(t-h)) l(x(t-h)) \\
& \leq \varepsilon_{2} x^{T}(t) P W W^{T} P x(t)+\varepsilon_{2}^{-1} x^{T}(t-h) G^{T} G x(t-h), \\
2 x^{T}(t) P(\Delta W) l(x(t-h)) & =2 x^{T}(t) P M F N_{2} l(x(t-h)) \\
& \leq \varepsilon_{3} x^{T}(t) P M M^{T} P x(t)+\varepsilon_{3}^{-1} l^{T}(x(t-h)) N_{2}^{T} N_{2} l(x(t-h)) \\
& \leq \varepsilon_{3} x^{T}(t) P M M^{T} P x(t)+\varepsilon_{3}^{-1} \lambda_{\max }\left(N_{2}^{T} N_{2}\right) l^{T}(x(t-h)) l(x(t-h)) \\
& \leq \varepsilon_{3} x^{T}(t) P M M^{T} P x(t)+\varepsilon_{3}^{-1} \lambda_{\max }\left(N_{2}^{T} N_{2}\right) x^{T}(t-h) G^{T} G x(t-h) .
\end{aligned}
$$

Note that (14) can be rewritten, by the Schur Complement Lemma (Lemma 3), as

$$
-P+\left(M^{T} P\right)^{T}\left(\varepsilon_{4} I\right)^{-1}\left(M^{T} P\right)<0,
$$

or

$$
P^{-1}-\varepsilon_{4}^{-1} M M^{T}>0 \text {. }
$$


Hence, it follows from Lemma 2 that

$$
\begin{aligned}
& {[(\Delta C) x(t)+(\Delta D) x(t-h)]^{T} P[(\Delta C) x(t)+(\Delta D) x(t-h)] } \\
= & \left\{M F\left[N_{3} x(t)+N_{4} x(t-h)\right]\right\}^{T} P\left\{M F\left[N_{3} x(t)+N_{4} x(t-h)\right]\right\} \\
\leq & \varepsilon_{4}\left[N_{3} x(t)+N_{4} x(t-h)\right]^{T}\left[N_{3} x(t)+N_{4} x(t-h)\right] .
\end{aligned}
$$

Furthermore, it can be seen from Lemma 4 that

$$
\int_{t-h}^{t} x^{T}(s) Q_{2} x(s) d s \geq h^{-1}\left(\int_{t-h}^{t} x(s) d s\right)^{T} Q_{2}\left(\int_{t-h}^{t} x(s) d s\right) .
$$

Define

$$
\zeta(t):=\left[\begin{array}{c}
x(t) \\
x(t-h) \\
\int_{t-h}^{t} x(s) d s
\end{array}\right], \Theta:=\left[\begin{array}{ccc}
\Omega_{1}+\Omega_{2} & \varepsilon_{4} N_{4}^{T} N_{3} & 0 \\
\varepsilon_{4} N_{3}^{T} N_{4} & \Xi & 0 \\
0 & 0 & -h^{-1} Q_{2}
\end{array}\right]
$$

where $\Omega_{1}, \Omega_{2}$ and $\Xi$ are defined in (9), (10) and (13), respectively.

It is obvious from (18) that $\Theta<0$. Also, there must exist a scalar $\alpha>0$ such that

$$
\Theta+\left[\begin{array}{ccc}
\alpha I & 0 & 0 \\
0 & 0 & 0 \\
0 & 0 & 0
\end{array}\right]<0
$$

Using (22)-(26), after tedious algebraic manipulations, we can obtain from (21) that

$$
\begin{aligned}
d V(t, x(t)) \leq & {\left[\begin{array}{c}
x(t) \\
x(t-h) \\
\int_{t-h}^{t} x(s) d s
\end{array}\right]^{T}\left[\begin{array}{ccc}
\Omega_{1}+\Omega_{2} & \varepsilon_{4} N_{4}^{T} N_{3} & 0 \\
\varepsilon_{4} N_{3}^{T} N_{4} & \Xi & 0 \\
0 & 0 & -h^{-1} Q_{2}
\end{array}\right]\left[\begin{array}{c}
x(t) \\
x(t-h) \\
\int_{t-h}^{t} x(s) d s
\end{array}\right] } \\
& +\left\{2 x^{T}(t) P[(\Delta C) x(t)+(\Delta D) x(t-h)]\right\} d w(t) \\
= & \zeta^{T}(t) \Theta \zeta(t) d t+\left\{2 x^{T}(t) P[(\Delta C) x(t)+(\Delta D) x(t-h)]\right\} d w(t) .
\end{aligned}
$$

Taking the mathematical expectation of both sides of (29) and considering (28), we have

$$
\frac{d \mathbb{E} V(t, x(t))}{d t} \leq \mathbb{E}\left(\zeta^{T}(t) \Theta \zeta(t)\right) \leq-\alpha \mathbb{E}|x(t)|^{2}
$$

which indicates from the Lyapunov stability theory that the dynamics of the neural network (5) is robustly, globally, asymptotically stable in the mean square. This completes the proof of Theorem 1.

Remark 3: Notice that in Theorem 1, the matrix inequalities (14)(15) are linear on the parameters $\mu_{i}>0$ $(i=1,2,3,4), P>0, Q_{1}>0$ and $Q_{2}>0$. Therefore, by using the Matlab LMI toolbox, it is straightforward to check the feasibility of $(14)(15)$ without tuning any parameters. Different from the existing results based on matrix norm computation, such as those given in [1], [6], the LMI approach developed in this paper is numerically more efficient [4]. The LMI Control Toolbox implements state-of-the-art interior-point LMI solvers. While these solvers are significantly faster than classical convex optimization algorithms, it should be kept in mind that the complexity of LMI computations remains higher than that of solving, say, a Riccati equation. For instance, problems with a thousand design variables typically take over an hour on today's 
workstations [4]. However, research on LMI optimization is a very active area in the applied math, optimization and the operations research community, and substantial speed-ups can be expected in the future.

Remark 4: Note that Lemma 1 is used in the proof of Theorem 1 to tackle the parameter uncertainties, hence certain conservatism might be introduced. Such conservatism can be significantly reduced by selecting proper scalar parameters $\varepsilon$, see [28] for more details. We like to point out that, following the similar line of [23], it is not difficult to prove the exponential stability (in the mean square) of the neural network (5) under same conditions in Theorem 1.

In the following, we show that our main results can be easily specialized to two cases that have been studied in the literature.

First, assume that there are no stochastic disturbances, and the neural network is described as

$$
\dot{x}(t)=-(A+\Delta A) x(t)+(W+\Delta W) l(x(t-h)) .
$$

We have the following results.

Corollary 1: If there exist positive scalars $\mu_{i}>0(i=1,2,3)$ and positive definite matrices $P=P^{T}>0$, $Q_{1}=Q_{1}^{T}>0$ and $Q_{2}=Q_{2}^{T}>0$ such that the following linear matrix inequality

$\left[\begin{array}{cccccccc}-A P-P A+Q_{1}+h Q_{2} & 0 & P M & \mu_{1} N_{1}^{T} & P W & P M & 0 & 0 \\ 0 & -Q_{1} & 0 & 0 & 0 & 0 & \mu_{2} G^{T} & \mu_{3} \lambda_{\max }^{1 / 2}\left(N_{2}^{T} N_{2}\right) G^{T} \\ M^{T} P & 0 & -\mu_{1} I & 0 & 0 & 0 & 0 & 0 \\ \mu_{1} N_{1} & 0 & 0 & -\mu_{1} I & 0 & 0 & 0 & 0 \\ W^{T} P & 0 & 0 & 0 & -\mu_{2} I & 0 & 0 & 0 \\ M^{T} P & 0 & 0 & 0 & 0 & -\mu_{3} I & 0 & 0 \\ 0 & \mu_{2} G & 0 & 0 & 0 & 0 & -\mu_{2} I & 0 \\ 0 & \mu_{3} \lambda_{\max }^{1 / 2}\left(N_{2}^{T} N_{2}\right) G & 0 & 0 & 0 & 0 & 0 & -\mu_{3} I\end{array}\right]<0$

hold, then the dynamics of the neural network (31) is robustly, globally, asymptotically stable.

Second, if there are no parameter uncertainties in $A$ and $W$, that is, the neural network is simplified to

$$
d x(t)=[-A x(t)+W l(x(t-h))] d t+[(\Delta C) x(t)+(\Delta D) x(t-h)] d w(t),
$$

then we have the following corollary.

Corollary 2: If there exist positive scalars $\mu_{1}>0, \mu_{2}>0$ and positive definite matrices $P=P^{T}>0$, $Q_{1}=Q_{1}^{T}>0$ and $Q_{2}=Q_{2}^{T}>0$ such that the following two linear matrix inequalities

$$
\begin{gathered}
{\left[\begin{array}{cc}
-P & P M \\
M^{T} P & -\mu_{2} I
\end{array}\right]<0,} \\
{\left[\begin{array}{cccc}
-A P-P A+Q_{1}+h Q_{2}+\mu_{2} N_{3}^{T} N_{3} & \mu_{2} N_{4}^{T} N_{3} & P W & 0 \\
\mu_{4} N_{3}^{T} N_{4} & -Q_{1}+\mu_{2} N_{4}^{T} N_{4} & 0 & \mu_{1} G^{T} \\
W^{T} P & 0 & -\mu_{1} I & 0 \\
0 & \mu_{1} G & 0 & -\mu_{1} I
\end{array}\right]<0}
\end{gathered}
$$

hold, then the dynamics of the neural network (5) is globally asymptotically stable in the mean square.

\section{NumERICAL EXAMPLES}

Two simple examples are presented here in order to illustrate the usefulness of our main results. Our aim is to examine the global asymptotic stability of a given delayed stochastic neural network. 
Example 1. In this example, we consider a two-neuron uncertain neural network (31) with time delays but without stochastic disturbances, where

$$
\begin{gathered}
A=\left[\begin{array}{cc}
1.2 & 0 \\
0 & 1.15
\end{array}\right], W=\left[\begin{array}{cc}
0.4 & -1 \\
-1.4 & 0.4
\end{array}\right], M=\left[\begin{array}{ll}
0.1 & 0.5 \\
0.5 & 0.3
\end{array}\right], \\
N_{1}=\left[\begin{array}{cc}
0.6 & 0 \\
0 & 0.6
\end{array}\right], N_{2}=\left[\begin{array}{cc}
0.2 & 0 \\
0 & 0.2
\end{array}\right], \quad G=\left[\begin{array}{cc}
0.5 & 0 \\
0 & 0.5
\end{array}\right], h=0.12
\end{gathered}
$$

By using the Matlab LMI toolbox, we solve the LMI (32) for $\mu_{i}>0(i=1,2,3), P>0, Q_{1}>0, Q_{2}>0$ and obtain

$$
\begin{gathered}
\mu_{1}=1.4449 e+008, \quad \mu_{2}=4.6044 e+008, \quad \mu_{3}=6.2341 e+008, \\
P=10^{8}\left[\begin{array}{ll}
2.2695 & 0.0935 \\
0.0935 & 1.5337
\end{array}\right], \quad Q_{1}=10^{8}\left[\begin{array}{ll}
1.8982 & 0.0772 \\
0.0772 & 1.2321
\end{array}\right], \quad Q_{2}=10^{-10}\left[\begin{array}{cc}
0.0004 & -0.0108 \\
-0.0108 & 0.1145
\end{array}\right] .
\end{gathered}
$$

Therefore, it follows from Corollary 1 that the two-neuron neural network (31) is robustly globally asymptotically stable.

Example 2. Now, let us consider a third-order delayed stochastic neural network (5) with parameter uncertainties. The network data are given as follows:

$$
\begin{gathered}
A=\left[\begin{array}{ccc}
2.2 & 0 & 0 \\
0 & 2.4 & 0 \\
0 & 0 & 2.6
\end{array}\right], W=\left[\begin{array}{ccc}
0.3 & -1.8 & 0.5 \\
-1.1 & 1.6 & 1.1 \\
0.6 & 0.4 & -0.3
\end{array}\right], \\
M=\operatorname{diag}\{0.1,0.5,0.3\}, \quad N_{1}=0.6 I_{3}, \quad N_{2}=N_{3}=N_{4}=0.2 I_{3}, \quad G=0.5 I_{3}, \quad h=0.8 .
\end{gathered}
$$

Again, by solving the LMIs (14)-(15) for $\mu_{i}>0(i=1,2,3,4), P>0, Q_{1}>0, Q_{2}>0$, we have

$$
\begin{gathered}
\mu_{1}=2.8220 e+008, \quad \mu_{2}=4.8203 e+008, \quad \mu_{3}=3.5802 e+008, \quad \mu_{4}=2.9091 e+008, \\
P=10^{8}\left[\begin{array}{cccc}
1.9536 & 0.3183 & 0.0282 \\
0.3183 & 1.6569 & -0.0041 \\
0.0282 & -0.0041 & 2.0321
\end{array}\right], \\
Q_{1}=10^{8}\left[\begin{array}{lll}
2.6542 & 0.6378 & 0.0860 \\
0.6378 & 2.3518 & 0.0310 \\
0.0860 & 0.0310 & 3.2208
\end{array}\right],
\end{gathered}
$$

which indicates from Theorem 1 that the delayed uncertain stochastic neural network (5) is robustly, globally, asymptotically stable in the mean square.

\section{Conclusions}

In this paper, we have dealt with the problem of global asymptotic stability analysis for a class of uncertain stochastic delayed neural networks, which involve both the parameter uncertainties and the time-delays. We have removed the traditional monotonicity and smoothness assumptions on the activation function. A linear matrix inequality (LMI) approach has been developed to derive the stability criteria, which can be tested easily using the Matlab LMI toolbox. Simple examples have been used to demonstrate the usefulness of the main results. One of the future research topics would be the extension of the present results to more general cases, for example, the case that the delays are mixed, including both discrete and distributed delays, and the case that exponential stability is investigated. The results will appear in the near future. 


\section{REFERENCES}

[1] S. Arik, Stability analysis of delayed neural networks. IEEE Transactions on Circuits Systems -I, vol. 47, pp. 1089-1092, 2000 .

[2] S. Arik, Global robust stability analysis of neural networks with discrete time delays, Chaos, Solitons and Fractals, vol. 26, no. 5, pp. 1407-1414, 2005.

[3] S. Blythe, X. Mao and X. Liao, Stability of stochastic delay neural networks, Journal of the Franklin Institute, vol. 338, pp. 481-495, 2001.

[4] S. Boyd, L. EI Ghaoui, E. Feron and V. Balakrishnan, Linear Matrix Inequalities in System and Control Theory. Philadelphia, PA: SIAM, 1994.

[5] T. Burton, Stability and periodic solution of ordinary differential equation and functional differential equations. Orlando, FL: Academic, 1985.

[6] J. Cao, Periodic oscillation and exponential stability of delayed CNNs, Physics Letters A, vol. 270, pp. 157-163, 2000.

[7] J. Cao, A. Chen and X. Huang, Almost periodic attractor of delayed neural networks with variable coefficients, Physics Letters A, vol. 340, pp. 104-120, 2005.

[8] J. Cao, D.-S. Huang and Y. Qu, Global robust stability of delayed recurrent neural networks, Chaos, Solitons and Fractals, vol. 23, pp. 221-229, 2005.

[9] J. Cao and D. W. C. Ho, A general framework for global asymptotic stability analysis of delayed neural networks based on LMI approach, Chalos, Solitons and Fractals, vol. 24, pp. 1317-1329, 2005.

[10] J. Cao and J. Wang, Global exponential stability and periodicity of recurrent neural networks with time delays, IEEE Trans. Circuits and Systems: Part I, vol. 52, no. 5, pp. 920-931, 2005.

[11] J. Cao and T. Chen, Globally exponentially robust stability and periodicity of delayed neural networks, Chaos, Solitons and Fractals, vol. 22, no. 4, pp. 957-963, 2004.

[12] A. Friedman, Stochastic differential equations and their applications, New York, Academic Press, 1976.

[13] P. Gahinet, A. Nemirovsky, A. J. Laub and M. Chilali, LMI Control Toolbox: For Use with Matlab. The Math Works, Inc. 1995.

[14] K. Gu, An integral inequality in the stability problem of time-delay systems. In: Proceedings of 39th IEEE Conference on Decision and Control, December 2000, Sydney, Australia, pp. 2805-2810, 2000.

[15] J. K. Hale, Theory of functional differential equations. New York: Springer-Verlag, 1977.

[16] J. J. Hopfield, Neural networks and physical systems with emergent collect computational abilities, Proc. Nat. Acad. Sci., USA, vol. 79, no. 2, pp. 2554-2558, 1982.

[17] J. J. Hopfield, Neurons with graded response have collective computational properties like those of two-state neurons, Proc. Nat. Acad. Sci., USA, vol. 81, pp. 3088-3092, 1984.

[18] J. Hu, S. Zhong and L. Liang, Exponential stability analysis of stochastic delayed cellular neural network, Chaos, Solitons 8 Fractals, in press, 2005.

[19] H. Huang, D. W. C. Ho and J. Lam, Stochastic stability analysis of fuzzy Hopfield neural networks with time-varying delays, IEEE Trans. Circuits and Systems: Part II, vol. 52, no. 5, pp. 251-255, May 2005.

[20] M. P. Joy, Results concerning the absolute stability of delayed neural networks, Neural Networks, vol. 13, pp. 613-616, 2000.

[21] G. Joya, M. A. Atencia and F. Sandoval, Hopfield neural networks for optimization: study of the different dynamics, Neurocomputing, vol. 43, pp. 219-237, 2002.

[22] W. J. Li and T. Lee, Hopfield neural networks for affine invariant matching, IEEE Trans. Neural Networks, vol. 12, pp. 14001410, 2001.

[23] Y. Liu, Z. Wang and X. Liu, Global exponential stability of generalized recurrent neural networks with discrete and distributed delays, Neural Networks, in press.

[24] L. Wan and J. Sun, Mean square exponential stability of stochastic delayed Hopfield neural networks, Physics Letters A, vol. 343, no. 4, pp. 306-318, 2005

[25] Y. Wang, L. Xie and C. E. de Souza, Robust control of a class of uncertain nonlinear systems, Systems 83 Control Letters, vol. 19, pp. 139-149, 1992.

[26] Z. Wang and H. Qiao, Robust filtering for bilinear uncertain stochastic discrete-time systems, IEEE Trans. Signal Processing, vol. 50, no. 3, pp. 560-567, 2002.

[27] Z. Wang, Y. Liu and X. Liu, On global asymptotic stability of neural networks with discrete and distributed delays, Physics Letters A, vol. 345, no. 4-6, pp. 299-308, 2005.

[28] L. Xie and Y. C. Soh, Robust Kalman filtering for uncertain systems, Systems E Control Letters, vol. 22, pp. 123-129, 1994. 
[29] S. Xu, J. Lam and D. W. C. Ho, Novel global robust stability criteria for interval neural networks with multiple time-varying delays, Physics Letters A, vol. 342, no. 4, pp. 322-330, 2005.

[30] S. Young, P. Scott and N. Nasrabadi, Object recognition using multilayer Hopfield neural network, IEEE Trans. Image Processing, vol. 6, no. 3, pp. 357-372, 1997.

[31] H. Zhao, Global asymptotic stability of Hopfield neural network involving distributed delays. Neural Networks, vol. 17, pp. 47-53, 2004. 